\title{
NASA launches wind-tunnel tests by Cray-2 supercomputer
}

\section{San Francisco}

A MILITARY brass band played and experimental aircraft circled as NASA (National Aeronautics and Space Administration) officials in California formally unveiled on 9 March the world's most powerful supercomputer complex for designing aircraft.

At a dedication at the NASA-Ames Research Center, 40 miles south of San Francisco, NASA chiefs hailed the Numerical Aerodynamic Simulation (NAS) system as ranking in importance with the first use of a wind tunnel in 1873 by Francis Herbert Wenham and the first flight of the Wright brothers in 1903.

For NASA, the new development is, at the least, a badly needed sign that the post-Challenger space agency has not entirely lost its knack as a pathfinder in advanced engineering. Among the most formidable tasks for the new facility will be the design of the $\mathrm{X}-30$ prototype for the National Aerospace Plane, a hypothetical hypersonic vehicle endorsed by the Reagan administration.

With NAS, aeronautical engineers expect for the first time to be able to derive

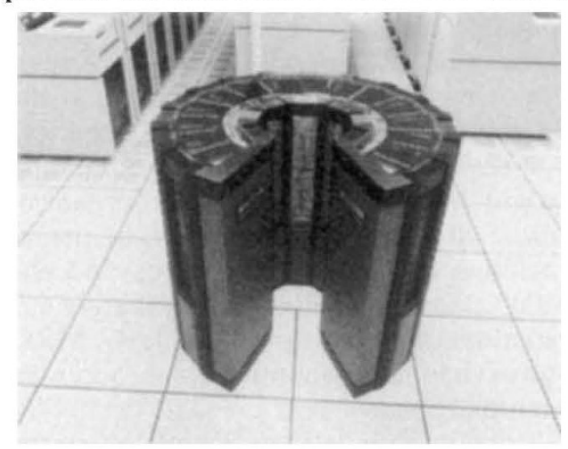

The NAS system's Cray-2 supercomputer

practically relevant solutions of the hydrodynamic equations for the flow of compressible viscous fluids developed more than a century ago and known as the Navier-Stokes equations. The solutions will be used as tools in roughing out designs of aircraft whose performance overwhelm ordinary computers, and which often cannot be tested even in the most powerful wind tunnels.

A Cray-2 supercomputer is, for the time being, the heart of NAS. The cooled machine is at least eight times faster than machines now used in computational aerodynamics and its memory is 40 times bigger. It is not the world's only Cray-2, but it has features giving it more versatility than any other.

In a year or so, NASA officials here expect to install a machine four times as fast. Front-runners for the sale are the still-embryonic Cray-3 or Cray YMP from Cray Research Corporation.
The NAS complex is the first routinely to use "Reynolds-averaged NavierStokes" equations to chart in threedimensional detail turbulent supersonic airflow over complex aircraft bodies. Its colourful animated displays can be commanded to appear at a score of workstations at Ames and at dozens of others linked by high-speed data links at other NASA centres, aerospace companies, defence laboratories and universities.

The powerful mathematics of fluid dynamics have previously been limited to highly idealized configurations, such as isolated and geometrically simple airfoils, and often to two dimensions. The NavierStokes equations have been called "innocuous in appearance . . marked by insidious pitfalls", with more than 60 partial derivative terms when expressed in three dimensions. The capacity of $10^{9}$ operations a second expected at NAS by 1989 should make it possible to calculate flow through a grid of a million points arrayed around aerodynamic shapes.

William F. Ballhaus $\mathrm{Jr}$, one of the originators of NAS, now says the facility took two major gambles. One was to order the Cray-2 in 1984 when NASA approved major spending, even though the machine did not then exist. The other was to settle on the AT\&T UNIX operating system, now a common protocol in managing computer networks but not when NAS was laid out. As the Ames team guessed right, the powerful machine has been easily incorporated in interactive networks among distant laboratories.

In spite of the new computer power, wind tunnels will continue to hold a vital place in aircraft design. Ballhaus says that "a computer can optimize the design, ...but to get its actual performance out to the third decimal place, we will still need to fly a real model in real air". And then, somebody must climb into a real airplane and take off in it.

\section{Soviet Academy of Sciences make room for next generation}

\section{London}

THE Soviet Academy of Sciences last week adopted a policy of "rejuvenation". In order to make room for younger talent, nobody over the age of 65 will normally be allowed to hold an administrative post in any of the institutes, laboratories or departments belonging to the academy - a ruling incidentally that bars 65 -year-old Andrei Sakharov from such a job. But superannuated academy scientists will be permitted, indeed encouraged, to devote their attention to training their successors. "In the interests of preserving intellectual potential", the new post of "honorary director" of an institute will be created, and a "staff of counsellors" made up of senior members of the academy is also envisaged.

The rejuvenation policy was formally presented and approved at the annual general meeting of the academy after several months of discussion. Last October, when Academician Vitalii Ginzburg, head of the I.E. Tamm Department of Theoretical Physics at the Lebedev Physics Institute reached his 70 th birthday, he published an article in the monthly Priroda advocating a policy of honourable retirement for academy scientists. The Soviet Academy has for years been heavily over-burdened with septuagenarians and octagenarians (in May 1985, according to Ginzburg's figures, more than 55 per cent of full members of the academy were over 70 , and 68.7 per cent over the new 65-year-old limit).

The academy, as the annual general meeting made clear, faces a massive efficiency drive. All fundamental science will be put on a footing of long-term forecasting and planning - a task that, Academy Vice-President Vladimiir Kotel'nikov noted, will have to be carried out soon and with limited resources. The academy presidium has defined 135 lines of research deemed to be of "immense significance to the national economy". Information science, the synthesis of new materials and scientific instrumentmaking are to receive priority attention, Kotel'nikov said, as "in these spheres difficulties arise over obtaining information from capitalist countries".

Academy departments and research establishments are to become more autonomous, which will free staff of much routine administrative work, but which will doubtless entail some interim confusion.

Scientific research in general must be made more "dynamic" and, Academy President Gurii Marchuk stressed, fundamental research must not simply support but must "overtake" the demands of technology and production. There is to be a major development of science in the east of the country, with the establishment of far eastern and Urals branches, which may well mean some scientists being encouraged to move eastwards.

In such an atmosphere of change and upheaval, many senior members and employees of the academy may well find honourable retirement a welcome option.

Vera Rich 